\title{
Laminins in Epithelial Cell Polarization: Old Questions in Search of New Answers
}

\author{
Karl S. Matlin, ${ }^{1}$ Satu-Marja Myllymäki, ${ }^{2}$ and Aki Manninen ${ }^{2}$ \\ ${ }^{1}$ Department of Surgery, The University of Chicago, Chicago, Illinois 60637-1470 \\ ${ }^{2}$ Biocenter Oulu, Oulu Center for Cell-Matrix Research, Faculty of Biochemistry and Molecular Medicine, \\ University of Oulu, Oulu 90220, Finland \\ Correspondence: kmatlin@uchicago.edu
}

Laminin, a basement membrane protein discovered in 1979, was shortly thereafter implicated in the polarization of epithelial cells in both mammals and a variety of lower organisms. To transduce a spatial cue to the intrinsic polarization machinery, laminin must polymerize into a dense network that forms the foundation of the basement membrane. Evidence suggests that activation of the small GTPase Rac1 by $\beta 1$-integrins mobilizes laminin-binding integrins and dystroglycan to consolidate formation of the laminin network and initiate rearrangements of both the actin and microtubule cytoskeleton to help establish the apicobasal axis. A key coordinator of spatial signals from laminin is the serine-threonine kinase Par-1, which is known to affect dystroglycan availability, microtubule and actin organization, and lumen formation. The signaling protein integrin-linked kinase (ILK) may also play a role. Despite significant advances, knowledge of the mechanism by which assembled laminin produces a spatial signal remains fragmentary, and much more research into the complex functions of laminin in polarization and other cellular processes is needed.

$T^{\mathrm{h}}$ he evolution of epithelial cells made multicellular organisms possible (Fahey and Degnan 2010; Leys and Riesgo 2011). Epithelial cells individually display a stable asymmetric organization or polarity, defined by a plasma membrane differentiated into domains consisting of a free or apical surface, a lateral surface, and a basal surface, each with a characteristic protein and lipid composition. Polarity extends as well to the cytoplasm, with organelles arranged along an axis running from the apical to basal surface. Most significantly, epithelial cells adhere to each other laterally and to an underlying extracellular matrix sheet known as the basement membrane to form a continuous, semipermeable cell layer or epithelium that shares the polarity of the individual cellular constituents. This combination of collective cell polarity and a barrier created by the epithelial layer divides multicellular organisms into compartments with different chemical compositions and specialized functions, and separates the inner milieu from the outside world.

Polarization of epithelial cells occurs through the cooperation of intrinsic and extrinsic polarization mechanisms (Nelson 2009). The intrinsic mechanism depends on mutually antagonistic interactions among a series of cy-

Editor: Keith E. Mostov

Additional Perspectives on Cell Polarity available at www.cshperspectives.org

Copyright (C) 2017 Cold Spring Harbor Laboratory Press; all rights reserved; doi: 10.1101/cshperspect.a027920

Cite this article as Cold Spring Harb Perspect Biol 2017;9:a027920 
K.S. Matlin et al.

toplasmic polarization signaling proteins commonly divided into three groups called the Par, Scribbled, and Crumbs complexes, and activation of the small GTPases Racl and Cdc42 (Nelson 2009). The extrinsic polarization mechanism, on the other hand, provides spatial orientation cues to the cell from the environment, triggering the asymmetric distribution and activation of the complexes that make up the intrinsic mechanism. In early embryos, primary spatial cues take a variety of forms. In Caenorhabditis elegans, for example, the sperm entry point provides the cue, whereas in Drosophila asymmetry is inherited epigenetically through the process of oogenesis (Deng and Ruohola-Baker 2000; Dawes and Munro 2011; Thompson 2012). In many, if not most, other cases involving polarization of epithelial cells, either initially during development or in adults following injuries that disrupt polarity, there is evidence that cell adhesion to both other cells and to the basement membrane (BM) protein laminin provide spatial cues.

Laminin was discovered by Rupert Timpl in 1979 during biochemical analysis of a matrixlike material secreted by the EHS mouse sarcoma (Timpl et al. 1979). When used for immunohistochemistry, specific antibodies against this protein showed that laminin is localized in the BMs underlying epithelia and surrounding nerves and muscle fibers. In the 1980s, Peter Ekblom implicated laminin in the differentiation and polarization of the primordial kidney epithelium from induced metanephric mesenchyme in the mouse (Ekblom et al. 1980; Klein et al. 1988). Since then, further research in mammals and lower organisms has consistently supported the idea that laminin facilitates epithelial polarization. How laminin accomplishes this remains, however, unclear.

In this article, we review the evidence that laminin plays a critical role in the polarization of epithelial cells. We first describe the complex laminin family and how laminins contribute to the assembly and overall structure of the $\mathrm{BM}$. We then focus on laminin receptors expressed in epithelial cells, including both integrins and dystroglycan, and on their atypical distributions and functions in epithelia. Finally, we present experimental evidence supporting laminin's role as an element of the extrinsic polarization mechanism. Along the way we will highlight issues with experimental approaches that have, in our estimation, limited progress in this important area.

\section{THE LAMININ FAMILY}

All bilaterians express laminins that have a canonical heterotrimeric structure consisting of $\alpha, \beta$, and $\gamma$ subunits assembled into a crossshaped molecule (Fig. 1) (Miner and Yurchenco 2004; Fahey and Degnan 2012). The amino-terminal parts of each subunit form the three arms

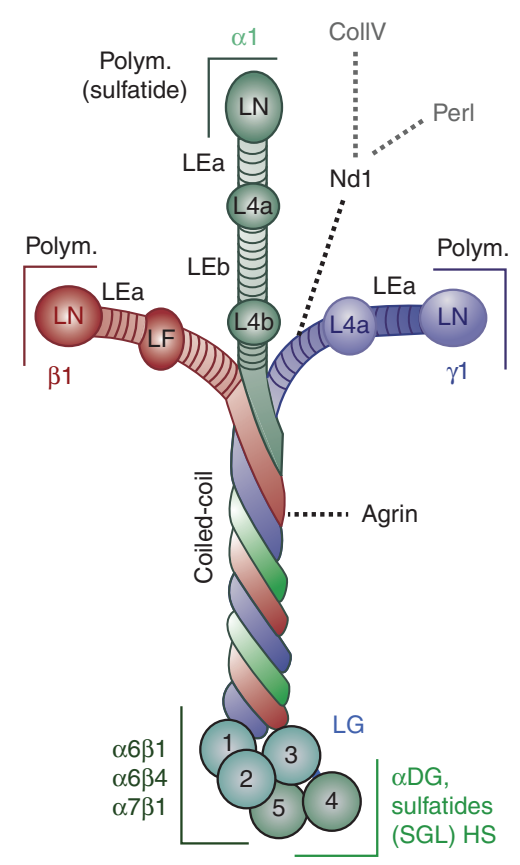

Figure 1. The structure of a canonical laminin molecule, using Lm111 as an example. Note the three laminin amino-terminal (LN) or polymerization domains on the $\alpha 1, \beta 1$, and $\gamma 1$ subunits, and the laminin globular (LG) domains on the carboxyl terminus of the $\alpha 1$ subunit. The binding sites of integrins, $\alpha$-dystroglycan ( $\alpha \mathrm{DG})$, and sulfated glycolipids (SGLs) as well heparan sulfates to LG domains are indicated. $\mathrm{Nd} 1$ refers to the binding site of nidogen, a protein that cross-links collagen type IV (CollIV) to laminin. (From Yurchenco 2015; reprinted, with permission, from Elsevier (C) 2015.) 
of the cross and the carboxy-terminal regions associate into the stem through coiled-coil interactions. The amino termini of all three chains are folded into homologous laminin amino-terminal (LN) domains. These mediate intermolecular interactions that drive the assembly of laminin networks and the formation of BMs. The carboxyl terminus of the $\alpha$ chain consists of a series of five laminin globular (LG) domains that mediate the interaction of laminin with cell surface receptors. Recent analysis indicates that even the sponge Amphimedon queenslandica has laminin-related genes whose products are theoretically capable of assembling into a cross-like structure similar to bilaterian laminin, linking the evolution of laminins to that of the earliest metazoans (Fahey and Degnan 2012). C. elegans and Drosophila, two invertebrate model organisms commonly used to study epithelial polarization, express the minimal set of conserved laminin chains, designated types $\alpha 1 / 2, \alpha 3 / 5, \beta$, and $\gamma$, and resulting, when assembled, in two different laminin molecules (Fahey and Degnan 2012). In mammals, the laminin family is expanded to five $\alpha$, four $\beta$, and three $\gamma$ chains, yielding at least 16 different forms. Four of the mammalian subunits, $\alpha 3 \mathrm{~A}$, $\alpha 4, \beta 3$, and $\gamma 2$, either arise from genes that are shortened relative to those coding for canonical subunits, or are truncated by alternative splicing or posttranslational processing. Because each of these shorter subunits lacks domains found in full-length laminin subunits, there are functional implications in cells expressing them that remain poorly understood (Miner and Yurchenco 2004; Yurchenco 2011, 2015; Fahey and Degnan 2012). In mammals, laminin names reflect their subunit composition such that laminin 511 (hereafter Lm511) is made up of $\alpha 5, \beta 1$, and $\gamma 1$, whereas Lm3A32 is composed of $\alpha 3 \mathrm{~A}, \beta 3$, and $\gamma 2$ (Aumailley et al. 2005). The predominant laminins expressed in mammalian epithelial cells are Lm111, Lm511, and Lm3A32.

Mammalian laminins with the canonical structure are capable of polymerizing into a network that forms the backbone of the BM (Yurchenco 2011, 2015). Based on experiments with cultured Schwann cells, the process of network assembly is believed to be initiated by the binding of individual laminin molecules to galactosyl-sulfatide, a membrane glycolipid, followed by recruitment of dystroglycan and integrins, other laminin receptors ( $\mathrm{Li}$ et al. 2005). As the concentration of bound laminin on the cell surface increases, individual laminin molecules associate with each other by formation of ternary complexes between LN domains from $\alpha, \beta$, and $\gamma$ subunits contributed by three different laminin molecules (Fig. 2). This loose laminin network is then stabilized by intercalation and assembly of a separate collagen IV network that is cross-linked to the laminin network by the protein nidogen. Other proteins, such as the proteoglycan perlecan, bind to the cell surface and insert themselves into the overall structure (Yurchenco 2011, 2015). In the absence of laminin, no assembly of collagen IV occurs; conversely, detectable BMs can be formed without collagen IV, consistent with a fundamental role for laminin ( $\mathrm{Li}$ et al. 2002; Poschl 2004). Nevertheless, the BMs assembled in the absence of collagen IV have structural abnormalities leading to functional defects in the epithelium (Poschl 2004). Moreover, in vitro studies hint that the assembly of collagen IV network contributes to regulation of epithelial polarity (Wang et al. 1990) (F Moafi, J Myllyharju, and A Manninen et al., unpubl.). As the BM forms, interaction of laminins with its cell surface receptors initiates intracellular signaling and alterations in the cytoskeleton (Colognato et al. 1999; Li et al. 2005).

The roles of laminin molecules lacking one or more LN domains in the regulation or modulation of BM assembly is not completely clear. Peter Yurchenco (2011) has suggested that laminins such as Lm411, which lack an $\alpha$ subunit LN domain, might form a loose network on the cell surface that is more dependent on the collagen IV network for its stability than one formed by typical laminins. In cell cultures of rat alveolar epithelial cells, Lm3A11, which also lacks the $\alpha$ LN domain, appears to assemble into fibrils, possibly driven by perlecan instead of or in addition to laminin-laminin interactions. Such structures may transduce mechanical signals (Jones et al. 2005; Urich et al. 2011). Lm3A32, which lacks both $\alpha$ and $\gamma$ LN do- 
K.S. Matlin et al.
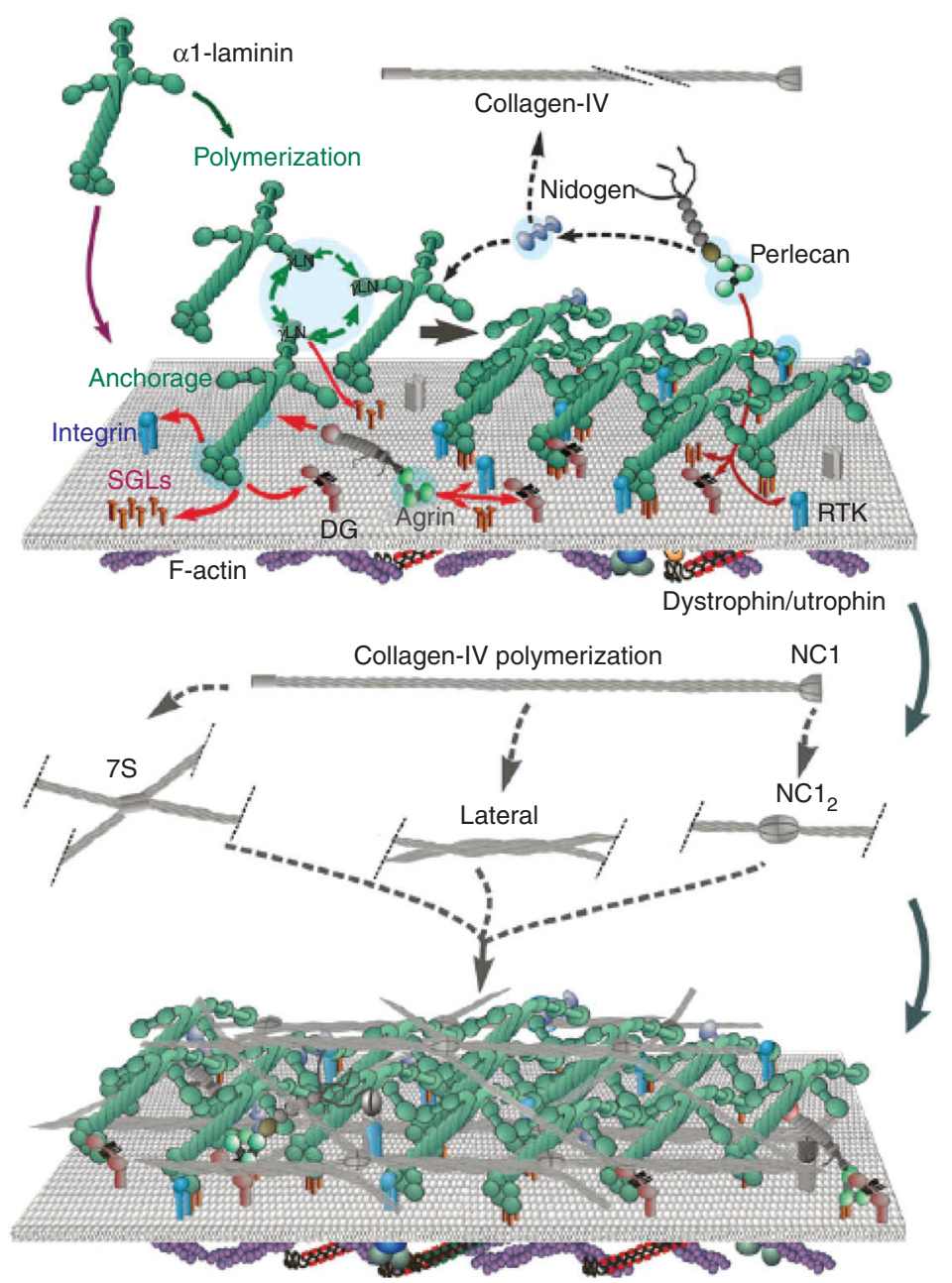

Figure 2. The current model for assembly of laminin and the basement membrane. According to this model (upper panel), individual laminin molecules bind to the cell surface by interacting with sulfated glycolipids (SGLs) via laminin globular (LG) domains and then polymerize through interactions of laminin amino-terminal (LN) domains contributed by three laminin molecules ( polymerization). This is followed by binding of dystroglycan (DG), integrin, and agrin (a heparan sulfate proteoglycan) to LG domains, initiating signaling through receptor tyrosine kinases (RTKs). In this version of the model, the $\alpha 1 \mathrm{LN}$ domain also binds to SGLs and integrins, causing the assembled laminin network to flatten against the membrane. After assembly of laminin, collagen IV molecules intercalate into the laminin network, associate with integrins, and polymerize (lower panel). The collagen network is cross-linked to the laminin network by nidogen. Perlecan (another heparan sulfate proteoglycan) and agrin also help cross-link collagen to laminin, thereby stabilizing the basement membrane. (From Yurchenco 2011; reprinted, with permission, from Cold Spring Harbor Laboratory Press (C) 2011.)

mains, coexists in the BMs of the epidermis and some other tissues with the canonical laminin Lm511 (as well as Lm3A11), where it is linked to the collagen IV network via nidogen (Tzu and Marinkovich 2008). The $\alpha 3 \mathrm{~A}$ and $\gamma 2$ subunits of Lm3A32 found in normal skin are proteo- lytically processed. On injury, however, both the skin and kidney tubular epithelial cells express unprocessed (but still genetically truncated) Lm3A32, which is thought to stimulate cell migration to close the wound (Zuk and Matlin 2002; Mak et al. 2006; Tzu and Marinkovich 
Laminin Polarization Cues

2008; Moyano et al. 2010; Greciano et al. 2012). Netrins, which are short, laminin-related proteins possessing LN-like domains, have also been reported to regulate BM assembly (Schneiders et al. 2007).

In Madin-Darby canine kidney (MDCK) cells, which form a continuous polarized epithelium in culture, the canonical laminin Lm511 is synthesized constitutively, whereas Lm3A32 is only expressed on disruption of the epithelium, consistent with a role for Lm3A32 in cell migration (Mak et al. 2006; Moyano et al. 2010; Greciano et al. 2012). Greciano et al. (2012) observed that the ability of MDCK cells to undergo directional migration was dependent on the ratio of deposited Lm511 and Lm3A32. They hypothesized that Lm3A32 interacts with and destabilizes the Lm511 network to generate a haptotactic gradient in the deposited extracellular matrix to facilitate directional migration, and speculated that effects on the Lm511 network might be mediated by the $\beta 3$ subunit of Lm3A32 (Greciano et al. 2012). The plausibility of this hypothesis is uncertain. Purified Lm111, which resembles Lm511, will assemble in solution in the presence of calcium in a manner analogous to network formation on the cell surface. Under these conditions, addition of increasing concentrations of Lm3A32 fails to inhibit Lm111 assembly, suggesting that there is no interaction between the two different laminins that blocks ternary complex formation (Yurchenco and Cheng 1994; Cheng et al. 1997). On the other hand, studies of molecular interactions between expressed LN domains from a variety of laminin subunits indicate that the $\beta 3 \mathrm{LN}$ domain found in $\mathrm{Lm} 3 \mathrm{~A} 32$ is capable of interacting with other LN domains (Odenthal et al. 2004). Furthermore, purified Lm3A32 disrupts a matrix of Lm111 assembled on a glass coverslip, as monitored by atomic force microscopy (Chiang et al. 2011). This potential modulation of network assembly by laminins like Lm3A32 is relevant to laminin's possible role in epithelial polarization because evidence exists that only assembled laminin can help to determine the apicobasal polarity axis (see below), and expression of Lm3A32 has been reported during polarization of
MDCK cell cysts (O’Brien et al. 2001; Monteleon et al. 2012).

\section{LAMININ RECEPTORS IN EPITHELIAL CELLS}

Integrins constitute the major family of cellular ECM receptors and many of the 24 known mammalian integrin $\alpha \beta$-heterodimers have been reported to bind to laminin (Horwitz et al. 1985; Languino et al. 1989; Ignatius et al. 1990; Sonnenberg et al. 1990; Goodman et al. 1991; Nishiuchi et al. 2006). Carboxy-terminal globular domains (particularly LG1-3) of the laminin $\alpha$ chain serve as a binding site for at least $\alpha 6 \beta 1$-, $\alpha 6 \beta 4$-, $\alpha 7 \beta 1$-, and $\alpha 3 \beta 1$-integrins (Kikkawa et al. 2000; Nishiuchi et al. 2006). Although the expression pattern of integrins is cell-type dependent, normal epithelial cells express several $\beta 1$-integrins (typically $\alpha 2 \beta 1$, $\alpha 3 \beta 1$, and $\alpha 6 \beta 1), \alpha 6 \beta 4$, and $\alpha \mathrm{V}$-integrins $(\alpha \mathrm{V} \beta 3, \alpha \mathrm{V} \beta 5, \alpha \mathrm{V} \beta 6$, and $\alpha \mathrm{V} \beta 8)$ (Schoenenberger et al. 1994; Howlett et al. 1995; Gilcrease 2007; Myllymäki et al. 2011; Teräväinen et al. 2013). The above-mentioned $\beta 1$-integrins and $\alpha 6 \beta 4$ all contribute to the establishment of apicobasal polarity, particularly in three-dimensional in vitro culture systems (Howlett et al. 1995; Lohikangas et al. 2001; Weaver et al. 2002; Yu et al. 2005; Myllymäki et al. 2011). Integrins link the extracellular matrix with the cellular actin cytoskeleton at integrin-linked adhesion complexes (ILACs; $\beta 1$ - and $\alpha \mathrm{V}$-integrins) and with intermediate filament networks at hemidesmosomes (HDs; $\alpha 6 \beta 4$ ), thereby forming a mechanical continuum connecting the ECM and epithelium (Kanchanawong et al. 2010; Nahidiazar et al. 2015). ILACs are not mere mechanical links as they form large multicomponent signaling platforms that regulate essentially all aspects of cell behavior (Geiger and Yamada 2011; Horton et al. 2015). However, mechanistic and proteomic studies of ILACs have been performed almost exclusively in fibroblasts seeded on fibronectin-coated substrates and, therefore, the function, dynamics, and composition of ILACs on laminin remains poorly characterized (Geiger and ZaidelBar 2012; Winograd-Katz et al. 2014; Horton et al. 2015). $\beta 1$ - and $\alpha 6 \beta 4$-integrins colocalize 
K.S. Matlin et al.

in laminin patches observed in polarized MDCK epithelial cells, presumably demarcating sites of cell-driven laminin assembly. Curiously, these laminin-rich patches are adjacent to, but do not colocalize with, ILAC markers such as talin, while they do overlap with an HD marker plectin (SM Myllymäki and A Manninen, unpubl.). HDs, in turn, are only found in epithelial cells and their assembly depends on the unique cytoplasmic tail of the integrin $\beta 4$ subunit, which supports interactions with the keratin cytoskeleton via the plakin family of cytoskeletal linker proteins (Walko et al. 2015). Intermediate filaments have unique mechanical properties but, unlike actin and microtubule polymers, do not possess intrinsic polarity (Goldman et al. 2008; Li and Gundersen 2008). Nevertheless, $\alpha 6 \beta 4$-integrins are involved in the regulation of apicobasal polarity, possibly by promoting $\mathrm{BM}$ assembly or by modifying outside in signaling from the laminin-rich ECM (Weaver et al. 2002; Myllymäki et al. 2011).

Dystroglycan, heparan-sulfate proteoglycans, and sulfated glycolipids all interact mainly with the LG4-5 domains of laminin $\alpha$ chains (Gee et al. 1993; Salmivirta et al. 1994; Ido 2004; Li et al. 2005). Similar to $\beta 1$-integrins, $\alpha \beta$-heterodimeric dystroglycan also connects with the actin cytoskeleton by interacting with utrophin and/or dystrophin, particularly in muscle tissues, where its main role is to strengthen the connection between the muscle cell cytoskeleton and the surrounding BM (Winder et al. 1995; Rybakova et al. 2000). Dystroglycan is ubiquitously expressed in epithelial cells. At least in epidermis, dystroglycan localizes to HDs, which strengthen the linkage between the epidermis and the underlying dermis (Herzog et al. 2004). However, dystroglycan also interacts with Par-1, component of the intrinsic polarization machinery, and might thereby regulate epithelial cell polarity and morphogenesis (Bello et al. 2015; Peng et al. 2015) (see discussion below).

\section{LAMININ'S ROLE IN POLARIZATION}

The first strong evidence that laminin is important for epithelial polarization was the report in
1988 that an antilaminin antibody blocked the differentiation and polarization of the primordial kidney epithelium in organ cultures of induced metanephric mesenchyme (Klein et al. 1988). This finding was later buttressed by the observation that an antibody against the $\alpha 6$ subunit of a laminin-binding integrin produced the same effect (Sorokin et al. 1990). At about the same time, a variety of other studies appeared, correlating the expression of laminin with epithelial differentiation and polarization (Leivo et al. 1980; Wang et al. 1990; Miner and Yurchenco 2004).

During early mouse development, laminin does not appear to be required for initial blastomere compaction and polarization at the eight-cell stage before implantation, consistent with first detection of all three laminin subunits at the 16-cell stage (Leivo et al. 1980; Fleming and Johnson 1988; Miner and Yurchenco 2004). Postimplantation studies based on homozygous knockout of the gene for the $\gamma 1$ subunit, a component of both Lm111 and Lm511, suggest that laminin is critical for both BM assembly and morphogenesis (Smyth et al. 1999; Miner and Yurchenco 2004).

Later work by $\mathrm{Li}$ and colleagues rigorously examined the role of laminin in epithelial polarization using mouse embryoid bodies as a model (Li et al. 2002, 2003). Embryoid bodies are derived from suspended mouse embryonic stem cells, and when cultured recapitulate endodermal and ectodermal differentiation (Murray and Edgar 2000; Li et al. 2003). Over a period of 6-7 days, as the endoderm forms, a BM assembles between the endoderm and primitive ectoderm. Through a process of cavitation and apoptosis, a lumen opens in the cyst-like structure and ectodermal cells polarize to produce an epiblast (Murray and Edgar 2000; Li et al. 2003). $\mathrm{Li}$ and colleagues (2002) used embryoid bodies null for the expression of either $\beta 1$-integrin, the laminin $\gamma 1$ subunit, or dystroglycan, to test the roles of laminin binding and assembly in epiblast differentiation. In the absence of $\beta 1$-integrin, neither epiblast differentiation nor BM assembly occurred; addition of exogenous laminin under these conditions was, however, able to drive both. Li et al. also observed that 
$\beta 1$-integrin-null epiblasts do not express the laminin $\alpha 1$ subunit, explaining the absence of endogenous assembled laminin in this mutant and indicating that $\beta 1$-integrin is not essential for anchorage of laminin to the cell surface in this model. Dystroglycan-null end-binding proteins (EBs) assembled laminin and had properly polarized epiblasts although their survival was affected. Addition of exogenous laminins unable to polymerize failed to stimulate differentiation of a polarized epiblast, strongly suggesting that laminin assembly into a network was critical for polarization (Li et al. 2002, 2003). Proper epiblast polarization in lamininsupplemented $\beta 1$-integrin and dystroglycannull EBs suggests that the basal polarity cue from laminin can be conveyed by multiple laminin receptors.

Studies in both Drosophila and C. elegans also support a role for laminin in epithelial differentiation and polarization. As mentioned previously, both organisms express two different laminin $\alpha$ subunits and single $\beta$ and $\gamma$ subunits (Fahey and Degnan 2012). Consequently, deletion or mutation of the $\beta$ subunit permits the roles of any possible laminin polymers to be probed. In Drosophila, mutation of the LanB1 gene prevented BM from forming, but did not inhibit embryogenesis. Nevertheless, organogenesis was generally abnormal with defects noted in adhesion, cell migration, and tubulogenesis (Urbano et al. 2009). Surprisingly, laminin was not required for gastrulation, leading the investigators to postulate that laminin is required only during epithelialization, which occurs after gastrulation in Drosophila (Urbano et al. 2009). In C. elegans, mutation of the $\beta$ subunit gene lam-1, prevented the polarization of pharyngeal precursor cells, as judged by apical localization of the polarity protein Par3. Laminin was, however, not universally required because the intestinal epithelium was able to differentiate and polarize in its absence (Rasmussen et al. 2012).

The linkage between epithelial polarization and laminin assembly was also shown in studies of MDCK cell cysts. When individual MDCK cells are suspended in a gel of type I, interstitial collagen, they proliferate to form three-dimen- sional polarized cysts with apical surfaces facing the lumen and basal surfaces facing the surrounding extracellular matrix (Fig. 3) (O'Brien et al. 2001; Yu et al. 2005). At the same time, a continuous BM composed of endogenous Lm511 and collagen IV, as well as other components, assembles on the outer surface of the cyst (O'Brien et al. 2001; Yu et al. 2005). In cells expressing a dominant negative $(\mathrm{dn})$ form of the small GTPase Rac1, or treated with a function-blocking antibody directed against $\beta 1$-integrin, the polarity of cells in the cyst is inverted and disorganized such that apical proteins now appear on the side of the cyst facing the extracellular matrix. At the same time, laminin and collagen IV still surround the cyst, but appear diffuse and disassembled (Fig. 3) (O'Brien et al. 2001; Yu et al. 2005). In cells expressing dnRac1, inverted polarity can be partially rescued by addition of exogenous Lm111 to the collagen gel (O'Brien et al. 2001). Inversion caused by anti$\beta 1$-integrin, on the other hand, can be rescued by expression of constitutively active Racl or by inhibition of the RhoA/ROCK1/myosin-II cascade (Yu et al. 2008). Significantly, these manipulations also rescue laminin assembly into a BM-like structure surrounding the cyst (Yu et al. 2005). The investigators of these studies suggest that an integrin containing the $\beta 1$ subunit, likely $\alpha 2 \beta 1$, interacts with collagen I molecules in the gel to activate Rac1, as supported also by more recent studies (Myllymäki et al. 2011; Teräväinen et al. 2013). Rac1 signaling, then, in some manner, facilitates laminin assembly resulting in the transmission of a spatial cue to cells that leads to the correct polar orientation (Yu et al. 2005). The role of Rac1 was challenged by a recent study on mammary epithelial cells in which mammary acinar morphogenesis was unaffected by deletion of Rac1, whereas $\beta 1$-integrins were still essential (Akhtar and Streuli 2013).

Another recent report relating MDCK cell cyst orientation and the integrity of the BM is generally consistent with the conclusions of the previously discussed studies, but also illustrates potential pitfalls of experimental systems used for three-dimensional culture. Monteleon et al. (2012) observed that MDCK cells grown in Matrigel, a commercial product derived from the 
K.S. Matlin et al.

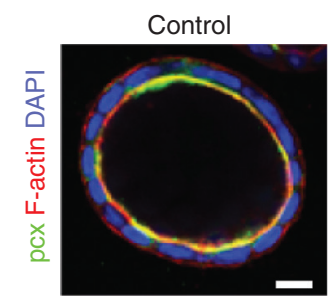

Control

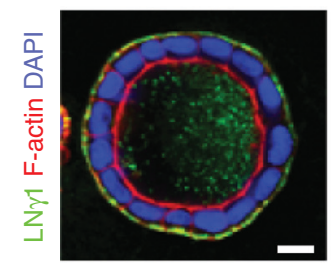

Control
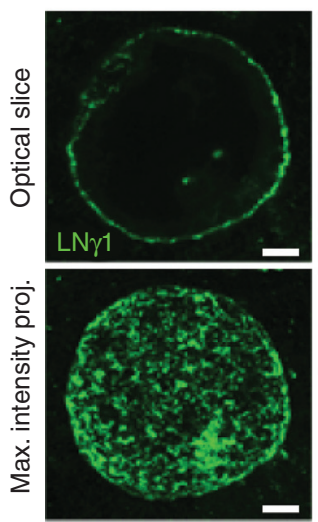

$\beta 1 K D$

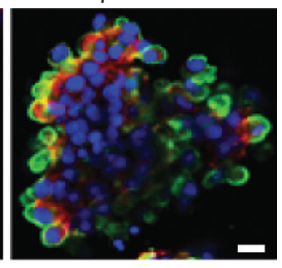

$\beta 1 \mathrm{KD}$
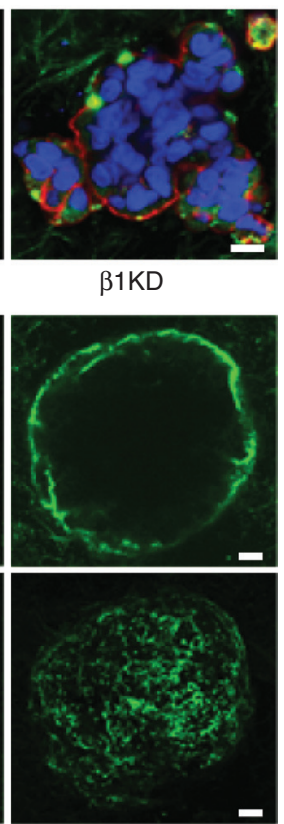

Figure 3. Laminin is deposited in a basement membrane-like pattern in three-dimensional cultures of Madin-Darby canine kidney (MDCK) epithelial cells. When MDCK cells are grown in collagen gels, they form polarized cysts with the apical surface facing the lumen of the cyst and the basal surface facing the collagen gel. Normal (control) cultures are shown on the left and MDCK cells in which expression of the $\beta 1$-integrin subunit (part of collagen and laminin receptors) is suppressed by siRNA are shown on the right. In controls, the apical protein podocalyxin $(\mathrm{pcx})$ and actin are detected on the apical surface by confocal fluorescence microscopy, whereas laminin, detected with an antibody to the $\gamma 1$ subunit (LN $\gamma 1$ ), forms a thin basal layer. The distribution of laminin is more easily seen in both an optical slice and a projection assembled from a through-focal series of optical slices in the two lower control panels. Knockdown of $\beta 1$-integrin disrupts overall cyst morphology and inverts polarity of actin and pcx, and disturbs the distribution of laminin (right panels) (SM Myllymäki and M Manninen, unpubl.; see also Yu et al. 2005). Scale bars, $10 \mu \mathrm{m}$.
Lm111 and collagen IV rich matrix of the EHSsarcoma, show inverted polarity when the expression of the Arf6 small-GTPase is suppressed with siRNA. They showed that this inversion is related to Racl inactivation, and is reversed by Racl activation, consistent with the findings of $\mathrm{Yu}, \mathrm{O}$ 'Brien, and colleagues (O'Brien et al. 2001; Yu et al. 2005, 2008). However, O'Brien et al. grew MDCK cell cysts in type I collagen gels, not Matrigel, and were able to rescue the inverted polarity phenotype by addition of Lm111 to the collagen gel. In the case of Monteleon et al. (2012) inversion that they link to Rac1 inactivation occurs even though MDCK cell cysts are immersed in the Lm111 provided by Matrigel, a discrepancy that they fail to note. Furthermore, they also observe the "assembly" of endogenous Lm332 (presumably Lm3A32) in normal MDCK cell cysts grown in Matrigel, as well as its lack of organization in inverted cysts, without accounting for the inability of Lm332 to assemble like other canonical laminins or the correlation of Lm332 expression in MDCK cells with loss of epithelial integrity (Cheng et al. 1997; Zuk and Matlin 2002; Mak et al. 2006; Moyano et al. 2010). More broadly considered, the use of Matrigel in three-dimensional cultures for experiments that probe the role of laminin and BM assembly in epithelial polarization is problematic. The presence of large amounts of Lm111 in Matrigel can potentially drive laminin assembly by mass action under circumstances when assembly of endogenous laminins might be inhibited, suppressing certain phenotypes and confounding interpretation of results. Furthermore, at least in the case of MDCK cells, $\mathrm{Lm} 511$, and not Lm111, is the normal canonical laminin that the cells express, and the naïve assumption that Lm111 can fully substitute for Lm511 may introduce further complications into experiments.

\section{HOW DOES LAMININ ACT AS A SPATIAL CUE?}

Based on the previous discussion, it seems likely that laminin acts as spatial cue during polarization of epithelial cells, but only when it is assembled, presumably into a structure involving 
ternary complexes between laminin molecules as found in authentic BMs. This raises two questions: First, is the assembly of laminin regulated by the cell, or is it driven solely by mass action when the density of laminin bound to the cell surface reaches a certain threshold? Second, once laminin is engaged with its receptors, how does it communicate with the polarization machinery to orient the cell?

A likely answer to the first question is that laminin assembly may initially be driven by mass action, but its transformation into a solid-state signaling platform appears to be regulated through engagement of laminin receptors with the actin cytoskeleton and the generation of specific signals. Work by Yurchenco has clearly shown that laminin assembly on cell surfaces can be driven by addition of exogenous molecules (Colognato et al. 1999; Li et al. 2005). In one case in particular, when exogenous laminin was added to myotubes capable of synthesizing only small amounts of endogenous laminin, it bound to the cell surface and was observed by light microscopy to transition from an initial aggregated, reticular distribution to a more focal, repeating polygonal pattern after more than 4 hours in culture (Colognato et al. 1999). Formation of the pattern on the cell surface required laminin polymerization, because it did not happen with a modified laminin incapable of polymerization, but was also dependent on actin and tyrosine phosphorylation because cytochalasin and a pan-tyrosine kinase inhibitor blocked the rearrangement. This suggests that conversion of the nascent laminin network into a more functional configuration is an active process (Colognato et al. 1999). The work of Li et al. (2005) further refined this sequence of events by demonstrating that laminin initially concentrates and begins to assemble on the cell surface by binding to sulfated glycolipids. Subsequently, integrin and dystroglycan receptors interact with the adherent laminin, and activate signaling through tyrosine kinases.

The answer to the second question likely involves the polarization protein Par-1 (Masuda-Hirata et al. 2009; Cohen et al. 2011; Yamahashi et al. 2011; Sato et al. 2013; Lewandowski and Piwnica-Worms 2014). Par-1 is a serine-threonine kinase identified through the same mutant screens of $C$. elegans that found other Par protein components of the intrinsic polarization machinery (Nelson 2009; Roignot et al. 2013). In polarized epithelial cells, Par-1 binds to the basolateral plasma membrane but is prevented from accumulating in the apical part of the cell by phosphorylation with atypical protein kinase $\mathrm{C}$ ( $\mathrm{PPKC}$ ), a component of the apical Par polarity complex (Hurov et al. 2004; Roignot et al. 2013). There is evidence that Par-1 regulates the formation of apical lumens in both MDCK cell monolayers overlaid with collagen and three-dimensional cyst cultures in collagen gels (Cohen et al. 2004, 2007, 2011; Lázaro-Diéguez et al. 2013). The level of Par-1 activity seems to be critical to this process, because overexpression of Par-1 can induce MDCK cells to form lateral, hepatocyte-like lumens instead of showing normal apicobasal polarized organization.

Most significantly, Par-1 appears to be involved in both laminin assembly and transduction of polarizing signals from the BM to the cell. Masuda-Hirata and colleagues found that Par-1 binds to the utrophin subunit of the dystroglycan laminin receptor (Masuda-Hirata et al. 2009; Yamashita et al. 2010). They further reported that Par-1 both regulates the localization of dystroglycan and the accumulation of laminin on the basal surface (Masuda-Hirata et al. 2009). Other, more recent, results are consistent with these observations (Lewandowski and Piwnica-Worms 2014). Cohen and colleagues observed that overexpression of Par-1 in MDCK cells affected the distribution of laminin on the basal surfaces of MDCK cell cysts in collagen gels (Cohen et al. 2011). In the latter case, this is likely related to Par-1 regulation of the actin cytoskeleton. Par-1 phosphorylates and inactivates insulin receptor tyrosine kinase substrate 53 (IRSp53), a protein responsible for mediating actin microfilament reorganization in response to Racl and cdc42, as well as guanine nucleotide exchange factor $\mathrm{H} 1$ (GEF-H1), which regulates RhoA-dependent actin cytoskeleton restructuring (Scita et al. 2008; Cohen et al. 2011; Yamahashi et al. 2011). Cohen et al. found that knockdown of IRSp53 mimicked the 
K.S. Matlin et al.

effects of Par-1 overexpression on laminin deposition (Cohen et al. 2011).

These results suggest a possible role for Par1 as a central modulator of spatial cues from laminin to the polarizing epithelial cell (Masuda-Hirata et al. 2009; Cohen et al. 2011; Lewandowski and Piwnica-Worms 2014). Not only is there evidence of Par-1 involvement in the activity and localization of laminin receptors, but also in the transduction of laminin signals through the cytoskeleton. In addition to regulating actin reorganization, Par-1 also alters microtubule dynamics through phosphorylation of microtubule-binding proteins, affecting the realignment of microtubules from a centrosomal focus to a vertical array that parallels the apicobasal axis in polarized cells (Cohen et al. 2004; Lázaro-Diéguez et al. 2013; Sato et al. 2013). As epithelial polarization proceeds, Par1 seems to balance the various factors transducing basal polarization signals-both too little and too much Par-1 activity can be disruptive to organization of the laminin matrix and downstream effects on polarization (MasudaHirata et al. 2009; Cohen et al. 2011).

The above data favor a role for dystroglycan in promoting laminin assembly yet do not exclude a role for integrins, particularly in conveying polarity signals once the laminin network has formed. Depletion of both $\beta 1$ - and $\beta 4$-integrins in MDCK cells (that still express dystroglycan) results in inverted polarity in $\mathrm{Ma}$ trigel-embedded MDCK cells in which abundant exogenous laminin is present (Myllymäki et al. 2011). Moreover, depletion of either the $\beta 1$ - or $\alpha 3$-integrin subunit interferes with lumen formation in Matrigel cultures.

Akhtar and colleagues reported that integrin-linked kinase (ILK), a pseudokinase adaptor protein that binds directly to the cytoplasmic tails of $\beta 1$ - and $\beta 3$-integrins, orchestrates endocytosis of apical membrane proteins away from the laminin-rich basal domain in mammary epithelial cells (Akhtar and Streuli 2013). ILK accomplished this by capturing microtubule plus ends at integrin adhesions via interactions with EBs, resulting in alignment of microtubules along the apicobasal polarity axis. In line with these data, ILK-null embryoid bodies had no defect in BM assembly, but the epiblast failed to polarize (Sakai et al. 2003).

\section{A WORKING MODEL}

The studies reviewed here suggest a model for how laminin provides a spatial cue to facilitate apicobasal polarization of epithelial cells (Fig. 4). When epithelial cells initially adhere to ECM proteins, whether collagen, laminin, or others, through engagement of integrin receptors, the small GTPase Racl is activated. As secreted laminin accumulates, it is initially concentrated on the cell surface by binding to sulfated glycolipids. The activities of Racl and Par-1, as well as signals from receptor tyrosine kinases, then mobilize both integrin and dystroglycan laminin receptors to reorganize and stabilize the nascent laminin network. At the same time, collagen IV and other components of the BM intercalate into the laminin network. Once engaged, signals from the laminin receptors, again modulated by Par- 1 together with ILK, lead to the reorganization of the microtubule and actin cytoskeletons to establish the apicobasal axis.

This model is consistent with observations that inhibition of Rac1 or blockade of $\beta 1$-integrins disrupts both laminin assembly and polarization (O'Brien et al. 2001; Yu et al. 2005), as well as a variety of findings linking Par-1 activity to apical lumen formation, actin and microtubule reorganization, and dystroglycan availability and activity (Cohen et al. 2004, 2007, 2011; Masuda-Hirata et al. 2009; Lázaro-Diéguez et al. 2013; Sato et al. 2013; Lewandowski and Piwnica-Worms 2014). The model presupposes the basal secretion of laminin, which may be under the control of regulators of membrane trafficking Crag and Rab10, and ultimately phosphoinositides, which are critical for epithelial polarization (Gassama-Diagne et al. 2006; Martin-Belmonte et al. 2007; Denef et al. 2008; Lerner et al. 2013; Devergne et al. 2014). What is not accounted for by this model is any possible role for nonpolymerizing laminins such as Lm3A32 in the regulation of laminin assembly (Odenthal et al. 2004; Chiang et al. 2011; Greciano et al. 2012). This laminin is expressed by epithelial cells like MDCK in a regulated fashion 


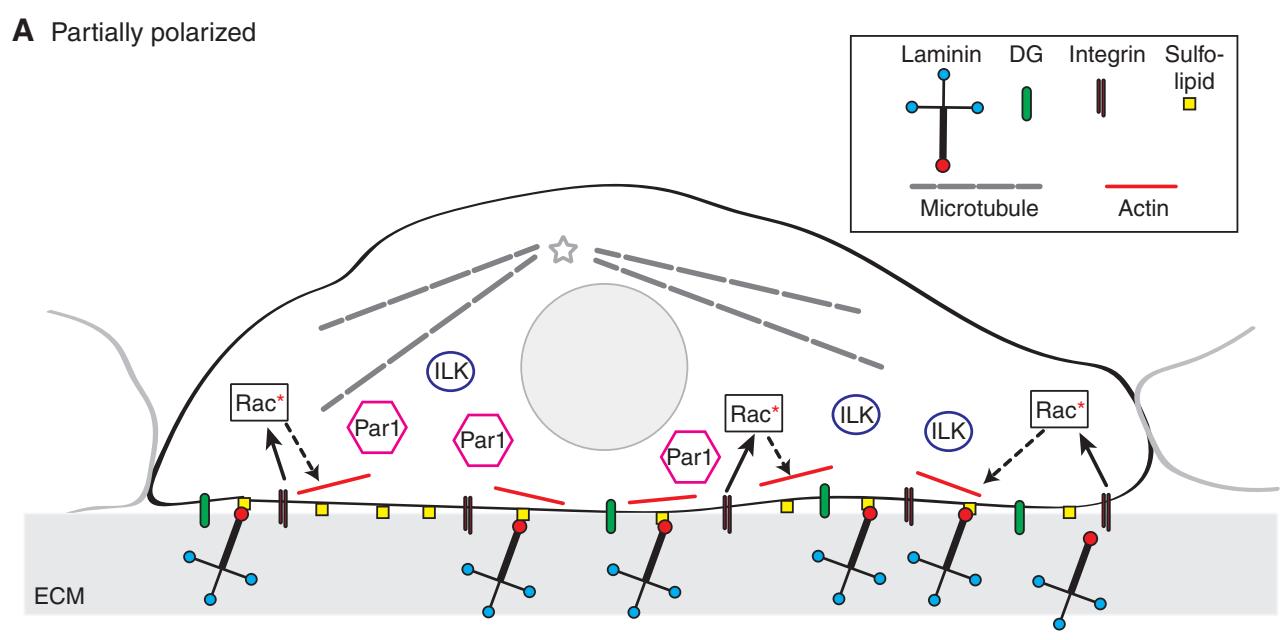

\section{B Polarized}

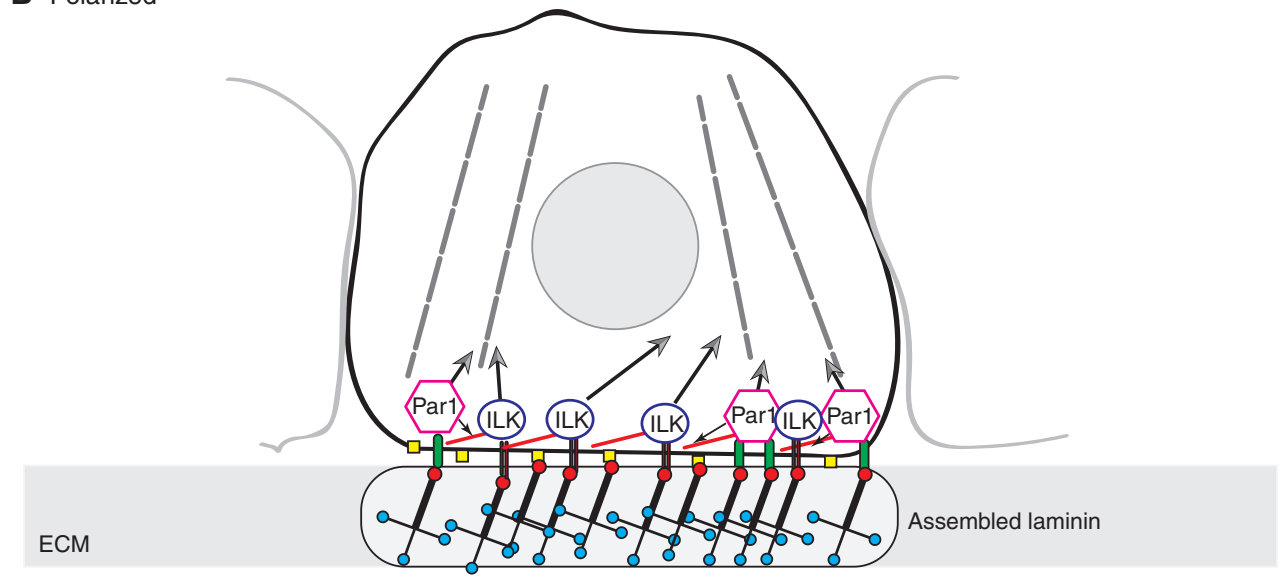

Figure 4. A preliminary model for the induction of epithelial polarity by laminin. Epithelial cells adhere to each other and extracellular matrix (ECM) proteins such as collagen and fibronectin through integrins, and activate Rac1 (Rac*), leading to alterations in cortical actin. Secreted laminin attaches to the basal surface via sulfated glycolipids. As the amount of laminin accumulates, it begins to polymerize and bind to integrins and dystroglycan (DG). The activities of Racl and Par-1, then mobilize both integrin and DG laminin receptors to reorganize and stabilize the nascent laminin network (assembled laminin). At the same time, collagen IV and other components of the basement membrane intercalate into the laminin network (not illustrated). Once engaged, signals from the laminin receptors, again modulated by Par- 1 together with integrin-linked kinase (ILK), lead to the reorganization of the microtubule and actin cytoskeletons to establish the apicobasal axis, leading to full polarization.

(Yu et al. 2005; Mak et al. 2006; Moyano et al. 2010), and is capable of disrupting laminin networks (Chiang et al. 2011), but its presence is often ignored by investigators in favor of a focus on single, canonical laminins.

Spatial signaling to epithelial cells from the extracellular matrix, like other aspects of epithe- lial polarization, is a robust property. Conditions of in vitro culture can circumvent or mask processes that are important in vivo, making signaling mechanisms from laminin hard to unravel. Furthermore, laminin molecules are difficult to study because of their large size and complex domain structure. Even with the 
K.S. Matlin et al.

many tour de force studies of Peter Yurchenco and his colleagues (McKee et al. 2009), the detailed organization, permeability, and mechanical properties of laminin networks and the overall BM are essentially unknown. Despite these many obstacles, more work to understand this essential, solid-state-signaling platform is justified and urgently needed.

\section{ACKNOWLEDGMENTS}

This article is dedicated to the memories of Elizabeth (Betty) Hay and Peter Ekblom, and to the many colleagues over the years that contributed to our work on integrins and laminins in epithelial cells. Research in the authors' laboratories was supported by grants from the $\mathrm{Na}$ tional Institutes of Health (K.S.M.) and Academy of Finland (140974, 263770, 135560; A.M. and S.-M.M.).

\section{REFERENCES}

Akhtar N, Streuli CH. 2013. An integrin-ILK-microtubule network orients cell polarity and lumen formation in glandular epithelium. Nat Cell Biol 15: 17-27.

Aumailley M, Bruckner-Tuderman L, Carter W, Deutzmann R, Edgar D, Ekblom P, Engel J, Engvall E, Hohenester E, Jones J, et al. 2005. A simplified laminin nomenclature. Matrix Biol 24: 326-332.

Bello V, Moreau N, Sirour C, Hidalgo M, Buisson N, Darribere T. 2015. The dystroglycan: Nestled in an adhesome during embryonic development. Dev Biol 401: 132-142.

Cheng Y, Champliaud M, Burgeson R, Marinkovich M, Yurchenco P. 1997. Self-assembly of laminin isoforms J Biol Chem 272: 31525-31532.

Chiang LY, Poole K, Oliveira BE, Duarte N, Sierra YAB, Bruckner-Tuderman L, Koch M, Hu J, Lewin GR. 2011. Laminin-332 coordinates mechanotransduction and growth cone bifurcation in sensory neurons. Nat Neurosci 14: $993-1000$.

Cohen D, Brennwald PJ, Rodriguez-Boulan E, Müsch A. 2004. Mammalian PAR-1 determines epithelial lumen polarity by organizing the microtubule cytoskeleton. J Cell Biol 164: 717-727.

Cohen D, Tian Y, Müsch A. 2007. Parlb promotes hepatictype lumen polarity in Madin Darby canine kidney cells via myosin II- and E-cadherin-dependent signaling. $\mathrm{Mol}$ Biol Cell 18: 2203-2215.

Cohen D, Fernandez D, Lázaro-Diéguez F, Müsch A. 2011. The serine/threonine kinase Parlb regulates epithelial lumen polarity via IRSp53-mediated cell-ECM signaling. J Cell Biol 192: 525-540.
Colognato H, Winkelmann D, Yurchenco P. 1999. Laminin polymerization induces a receptor cytoskeleton network. J Cell Biol 145: 619-631.

Dawes AT, Munro EM. 2011. PAR-3 oligomerization may provide an actin-independent mechanism to maintain distinct par protein domains in the early Caenorhabditis elegans embryo. Biophys J 101: 1412-1422.

Denef N, Chen Y, Weeks SD, Barcelo G, Schüpbach T. 2008. Crag regulates epithelial architecture and polarized deposition of basement membrane proteins in Drosophila. Dev Cell 14: 354-364.

Deng WM, Ruohola-Baker H. 2000. Laminin A is required for follicle cell-oocyte signaling that leads to establishment of the anterior-posterior axis in Drosophila. Curr Biol 10: 683-686.

Devergne O, Tsung K, Barcelo G, Schüpbach T. 2014. Polarized deposition of basement membrane proteins depends on phosphatidylinositol synthase and the levels of phosphatidylinositol 4,5-bisphosphate. Proc Natl Acad Sci 111: 7689-7694.

Ekblom P, Alitalo K, Vaheri A, Timpl R, Saxen L. 1980. Induction of a basement membrane glycoprotein in embryonic kidney: Possible role of laminin in morphogenesis. Proc Natl Acad Sci 77: 485-489.

Fahey B, Degnan BM. 2010. Origin of animal epithelia: Insights from the sponge genome. Evol Dev 12: 601-617.

Fahey B, Degnan BM. 2012. Origin and evolution of laminin gene family diversity. Mol Biol Evol 29: 1823-1836.

Fleming TP, Johnson MH. 1988. From egg to epithelium. Annu Rev Cell Biol 4: 459-485.

Gassama-Diagne A, Yu W, ter Beest M, Martin-Belmonte F, Kierbel A, Engel J, Mostov K. 2006. Phosphatidylinositol3,4,5-trisphosphate regulates the formation of the basolateral plasma membrane in epithelial cells. Nat Cell Biol 8: $963-970$.

Gee SH, Blacher RW, Douville PJ, Provost PR, Yurchenco PD, Carbonetto S. 1993. Laminin binding protein 120 from brain is closely related to the dystrophin-associated glycoprotein, dystroglycan, and binds with high affinity to the major heparin binding domain of laminin. J Biol Chem 268: 14972-14980.

Geiger B, Yamada KM. 2011. Molecular architecture and function of matrix adhesions. Cold Spring Harb Perspect Biol 3: a005033.

Geiger T, Zaidel-Bar R. 2012. Opening the floodgates: Proteomics and the integrin adhesome. Curr Opin Cell Biol 24: $562-568$.

Gilcrease MZ. 2007. Integrin signaling in epithelial cells. Cancer Lett 247: 1-25.

Goldman RD, Grin B, Mendez MG, Kuczmarski ER. 2008. Intermediate filaments: Versatile building blocks of cell structure. Curr Opin Cell Biol 20: 28-34.

Goodman SL, Aumailley M, von der Mark H. 1991. Multiple cell surface receptors for the short arms of laminin: $\alpha 1 \beta 1$ integrin and RGD-dependent proteins mediate cell attachment only to domains III in murine tumor laminin. J Cell Biol 113: 931-941.

Greciano PG, Moyano JV, Buschmann MM, Tang J, Lu Y, Rudnicki J, Manninen A, Matlin KS. 2012. Laminin 511 partners with laminin 332 to mediate directional migra- 
tion of Madin-Darby canine kidney epithelial cells. Mol Biol Cell 23: 121-136.

Herzog C, Has C, Franzke CW, Echtermeyer FG, SchlötzerSchrehardt U, Kräger S, Gustafsson E, Fässler R, Bruckner-Tuderman L. 2004. Dystroglycan in skin and cutaneous cells: $\beta$ subunit is shed from the cell surface. J Invest Dermatol 122: $1372-1380$.

Horton ER, Byron A, Askari JA, Ng DHJ, Millon-Frémillon A, Robertson J, Koper EJ, Paul NR, Warwood S, Knight D, et al. 2015. Definition of a consensus integrin adhesome and its dynamics during adhesion complex assembly and disassembly. Nat Cell Biol 17: 1577-1587.

Horwitz A, Duggan K, Greggs R, Decker C, Buck C. 1985. The cell substrate attachment (CSAT) antigen has properties of a receptor for laminin and fibronectin. J Cell Biol 101: $2134-2144$.

Howlett AR, Bailey N, Damsky C, Petersen OW, Bissell MJ. 1995. Cellular growth and survival are mediated by $\beta 1$ integrins in normal human breast epithelium but not in breast carcinoma. J Cell Sci 108: 1945-1957.

Hurov JB, Watkins JL, Piwnica-Worms H. 2004. Atypical PKC phosphorylates PAR-1 kinases to regulate localization and activity. Curr Biol 14: 736-741.

Ido H. 2004. Molecular dissection of the $\alpha$-dystroglycanand integrin-binding sites within the globular domain of human laminin-10. J Biol Chem 279: 10946-10954.

Ignatius MJ, Large TH, Houde M, Tawil JW, Barton A, Esch F, Carbonetto S, Reichardt LF. 1990. Molecular cloning of the rat integrin $\alpha 1$-subunit: A receptor for laminin and collagen. J Cell Biol 111: 709-720.

Jones JCR, Lane K, Hopkinson SB, Lecuona E, Geiger RC, Dean DA, Correa-Meyer E, Gonzales M, Campbell K, Sznajder JI, et al. 2005. Laminin-6 assembles into multimolecular fibrillar complexes with perlecan and participates in mechanical-signal transduction via a dystroglycan-dependent, integrin-independent mechanism. J Cell Sci 118: $2557-2566$.

Kanchanawong P, Shtengel G, Pasapera AM, Ramko EB, Davidson MW, Hess HF, Waterman CM. 2010. Nanoscale architecture of integrin-based cell adhesions. Nature 468: $580-584$.

Kikkawa Y, Sanzen N, Fujiwara H, Sonnenberg A, Sekiguchi K. 2000. Integrin binding specificity of laminin-10/11: Laminin-10/11 are recognized by $\alpha 3 \beta 1, \alpha 6 \beta 1$ and $\alpha 6 \beta 4$ integrins. J Cell Sci 113: 869-876.

Klein G, Langegger M, Timpl R, Ekblom P. 1988. Role of laminin $\alpha$ chain in the development of epithelial cell polarity. Cell 55: 331-341.

Languino LR, Gehlsen KR, Wayner E, Carter WG, Engvall E, Ruoslahti E. 1989. Endothelial cells use $\alpha 2 \beta 1$ integrin as a laminin receptor. J Cell Biol 109: 2455-2462.

Lázaro-Diéguez F, Cohen D, Fernandez D, Hodgson L, van IJzendoorn SCD, Müsch A. 2013. Par1b links lumen polarity with LGN-NuMA positioning for distinct epithelial cell division phenotypes. J Cell Biol 203: 251-264.

Leivo I, Vaheri A, Timpl R, Wartiovaara J. 1980. Appearance and distribution of collagens and laminin in the early mouse embryo. Dev Biol 76: 100-114.

Lerner DW, McCoy D, Isabella AJ, Mahowald AP, Gerlach GF, Chaudhry TA, Horner DS. 2013. A Rab10-dependent mechanism for polarized basement membrane secretion during organ morphogenesis. Dev Cell 24: 159-168.

Lewandowski KT, Piwnica-Worms H. 2014. Phosphorylation of the E3 ubiquitin ligase RNF41 by the kinase Par$1 \mathrm{~b}$ is required for epithelial cell polarity. J Cell Sci 127: 315-327.

Leys SP, Riesgo A. 2011. Epithelia, an evolutionary novelty of metazoans. J Exp Zool B Mol Dev Evol 318: 438-447.

Li R, Gundersen GG. 2008. Beyond polymer polarity: How the cytoskeleton builds a polarized cell. Nat Rev Mol Cell Biol 9: 860-873.

Li S, Harrison D, Carbonetto S, Fassler R, Smyth N, Edgar D, Yurchenco P. 2002. Matrix assembly, regulation, and survival functions of laminin and its receptors in embryonic stem cell differentiation. J Cell Biol 157: 1279-1290.

Li S, Edgar D, Fassler R, Wadsworth W, Yurchenco P. 2003. The role of laminin in embryonic cell polarization and tissue organization. Dev Cell 4: 613-624.

Li S, Liquari P, McKee K, Harrison D, Patel R, Lee S, Yurchenco P. 2005. Laminin-sulfatide binding initiates basement membrane assembly and enables receptor signaling in Schwann cells and fibroblasts. J Cell Biol 169: 179-189.

Lohikangas L, Gullberg D, Johansson S. 2001. Assembly of laminin polymers is dependent on $\beta 1$-integrins. Exp Cell Res 265: 135-144.

Mak GZ, Kavanaugh GM, Buschmann MM, Stickley SM, Koch M, Goss KH, Waechter H, Zuk A, Matlin KS. 2006. Regulated synthesis and functions of laminin 5 in polarized Madin-Darby canine kidney epithelial cells. Mol Biol Cell 17: 3664-3677.

Martin-Belmonte F, Gassama A, Datta A, Yu W, Rescher U, Gerke V, Mostov K. 2007. PTEN mediated apical segregation of phosphoinositides controls epithelial morphogenesis through Cdc42. Cell 128: 383-397.

Masuda-Hirata M, Suzuki A, Amano Y, Yamashita K, Ide M, Yamanaka T, Sakai M, Imamura M, Ohno S. 2009. Intracellular polarity protein PAR-1 regulates extracellular laminin assembly by regulating the dystroglycan complex. Genes Cells 14: 835-850.

McKee K, Capizzi S, Yurchenco P. 2009. Scaffold-forming and adhesive contributions of synthetic laminin-binding proteins to basement membrane assembly. J Biol Chem 284: 8984-8994.

Miner JH, Yurchenco PD. 2004. Laminin functions in tissue morphogenesis. Annu Rev Cell Dev Biol 20: 255-284.

Monteleon CL, Sedgwick A, Hartsell A, Dai M, Whittington C, Voytik-Harbin S, D'Souza-Schorey C. 2012. Establishing epithelial glandular polarity: Interlinked roles for ARF6, Rac1, and the matrix microenvironment. Mol Biol Cell 23: 4495-4505.

Moyano JV, Greciano PG, Buschmann MM, Koch M, Matlin KS. 2010. Autocrine transforming growth factor- $\beta 1$ activation mediated by integrin $\alpha \mathrm{V} \beta 3$ regulates transcriptional expression of laminin-332 in Madin-Darby canine kidney epithelial cells. Mol Biol Cell 21: 3654-3668.

Murray P, Edgar D. 2000. Regulation of programmed cell death by basement membranes in embryonic development. J Cell Biol 150: 1215-1221.

Myllymäki SM, Teräväinen TP, Manninen A. 2011. Two distinct integrin-mediated mechanisms contribute to apical lumen formation in epithelial cells. PLoS ONE 6: e19453. 
K.S. Matlin et al.

Nahidiazar L, Kreft M, van den Broek B, Secades P, Manders EMM, Sonnenberg A, Jalink K. 2015. The molecular architecture of hemidesmosomes, as revealed with superresolution microscopy. J Cell Sci 128: 3714-3719.

Nelson WJ. 2009. Remodeling epithelial cell organization: Transitions between front-rear and apical-basal polarity. Cold Spring Harb Perspect Biol 1: a000513.

Nishiuchi R, Takagi J, Hayashi M, Ido H, Yagi Y, Sanzen N, Tsuji T, Yamada M, Sekiguchi K. 2006. Ligand-binding specificities of laminin-binding integrins: A comprehensive survey of laminin-integrin interactions using recombinant $\alpha 3 \beta 1, \alpha 6 \beta 1, \alpha 7 \beta 1$ and $\alpha 6 \beta 4$ integrins. $M a$ trix Biol 25: 189-197.

O'Brien LE, Jou TS, Pollack AL, Zhang Q, Hansen SH, Yurchenco P, Mostov KE. 2001. Racl orientates epithelial apical polarity through effects on basolateral laminin assembly. Nat Cell Biol 3: 831-838.

Odenthal U, Haehn S, Tunggal P, Merkl B, Schomburg D, Frie C, Paulsson M, Smyth N. 2004. Molecular analysis of laminin N-terminal domains mediating self-interactions. J Biol Chem 279: 44504-44512.

Peng J, Awad A, Sar S, Hamze Komaiha O, Moyano R, Rayal A, Samuel D, Shewan A, Vanhaesebroeck B, Mostov K, et al. 2015. Phosphoinositide 3-kinase $\mathrm{p} 110 \delta$ promotes lumen formation through the enhancement of apico-basal polarity and basal membrane organization. Nat Commun 6: 5937.

Poschl E. 2004. Collagen IV is essential for basement membrane stability but dispensable for initiation of its assembly during early development. Development 131: 16191628.

Rasmussen JP, Reddy SS, Priess JR. 2012. Laminin is required to orient epithelial polarity in the C. elegans pharynx. Development 139: 2050-2060.

Roignot J, Peng X, Mostov K. 2013. Polarity in mammalian epithelial morphogenesis. Cold Spring Harb Perspect Biol 5: a013789.

Rybakova IN, Patel JR, Ervasti JM. 2000. The dystrophin complex forms a mechanically strong link between the sarcolemma and costameric actin. J Cell Biol 150: 12091214.

Sakai T, Li S, Docheva D, Grashoff C, Sakai K, Kostka G, Braun A, Pfeifer A, Yurchenco PD, Fässler R. 2003. Integrin-linked kinase (ILK) is required for polarizing the epiblast, cell adhesion, and controlling actin accumulation. Genes Dev 17: 926-940.

Salmivirta M, Mali M, Heino J, Hermonen J, Jalkanen M. 1994. A novel laminin-binding form of syndecan-1 (cell surface proteoglycan) produced by syndecan-1 cDNAtransfected NIH-3T3 cells. Exp Cell Res 215: 180-188.

Sato Y, Akitsu M, Amano Y, Yamashita K, Ide M, Shimada K, Yamashita A, Hirano H, Arakawa N, Maki T, et al. 2013. The novel PAR-1-binding protein MTCL1 has crucial roles in organizing microtubules in polarizing epithelial cells. J Cell Sci 126: 4671-4683.

Schneiders F, Maertens B, Bose K, Li Y, Brunken W, Paulsson M, Smyth N, Koch M. 2007. Binding of netrin-4 to laminin short arms regulates basement membrane assembly. J Biol Chem 282: 23750-23758.

Schoenenberger CA, Zuk A, Zinkl GM, Kendall D, Matlin KS. 1994. Integrin expression and localization in normal
MDCK cells and transformed MDCK cells lacking apical polarity. J Cell Sci 107: 527-541.

Scita G, Confalonieri S, Lappalainen P, Suetsugu S. 2008. IRSp53: Crossing the road of membrane and actin dynamics in the formation of membrane protrusions. Trends Cell Biol 18: 52-60.

Smyth N, Vatansever HS, Murray P, Meyer M, Frie C, Paulsson M, Edgar D. 1999. Absence of basement membranes after targeting the LAMC1 gene results in embryonic lethality due to failure of endoderm differentiation. J Cell Biol 144: 151-160.

Sonnenberg A, Linders CJ, Modderman PW, Damsky CH, Aumailley M, Timpl R. 1990. Integrin recognition of different cell-binding fragments of laminin (P1, E3, E8) and evidence that $\alpha 6 \beta 1$ but not $\alpha 6 \beta 4$ functions as a major receptor for fragment E8. J Cell Biol 110: 2145-2155.

Sorokin L, Sonnenberg A, Aumailley M, Timpl R, Ekblom P. 1990. Recognition of the laminin E8 cell-binding site by an integrin possessing the $\alpha 6$ subunit is essential for epithelial polarization in developing kidney tubules. J Cell Biol 111: 1265-1273.

Teräväinen TP, Myllymäki SM, Friedrichs J, Strohmeyer N, Moyano JV, Wu C, Matlin KS, Muller DJ, Manninen A. 2013. $\alpha \mathrm{V}$-integrins are required for mechanotransduction in MDCK epithelial cells. PLoS ONE 8: e71485.

Thompson BJ. 2012. Cell polarity: Models and mechanisms from yeast, worms and flies. Development 140: 13-21.

Timpl R, Rohde H, Robey PG, Rennard SI, Foidart JM, Martin GR. 1979. Laminin-A glycoprotein from basement membranes. J Biol Chem 254: 9933-9937.

Tzu J, Marinkovich MP. 2008. Bridging structure with function: Structural, regulatory, and developmental role of laminins. Int J Biochem Cell Biol 40: 199-214.

Urbano J, Torgler C, Molnar C, Tepass U, López-Varea A, Brown N, de Celis J, Martín-Bermudo M. 2009. Drosophila laminins act as key regulators of basement membrane assembly and morphogenesis. Development 136: 4165-4176.

Urich D, Eisenberg JL, Hamill KJ, Takawira D, Chiarella SE, Soberanes S, Gonzalez A, Koentgen F, Manghi T, Hopkinson SB, et al. 2011. Lung-specific loss of the laminin $\alpha 3$ subunit confers resistance to mechanical injury. J Cell Sci 124: 2927-2937.

Walko G, Castañón MJ, Wiche G. 2015. Molecular architecture and function of the hemidesmosome. Cell Tissue Res 360: $363-378$.

Wang AZ, Ojakian GK, Nelson WJ. 1990. Steps in the morphogenesis of a polarized epithelium. I: Uncoupling the roles of cell-cell and cell-substratum contact in establishing plasma membrane polarity in multicellular epithelial (MDCK) cysts. J Cell Sci 95: 137-151.

Weaver VM, Lelièvre S, Lakins JN, Chrenek MA, Jones JCR, Giancotti F, Werb Z, Bissell MJ. 2002. $\beta 4$ integrin-dependent formation of polarized three-dimensional architecture confers resistance to apoptosis in normal and malignant mammary epithelium. Cancer Cell 2: 205-216.

Winder SJ, Gibson TJ, Kendrick-Jones J. 1995. Dystrophin and utrophin: The missing links! FEBS Lett 369: 27-33. 
Winograd-Katz SE, Fässler R, Geiger B, Legate KR. 2014. The integrin adhesome: From genes and proteins to human disease. Nat Rev Mol Cell Biol 15: 273-288.

Yamahashi Y, Saito Y, Murata-Kamiya N, Hatakeyama M. 2011. Polarity-regulating kinase partitioning-defective 1b (PAR1b) phosphorylates guanine nucleotide exchange factor H1 (GEF-H1) to regulate RhoA-dependent actin cytoskeletal reorganization. J Biol Chem 286: 44576-44584.

Yamashita K, Suzuki A, Satoh Y, Ide M, Amano Y, MasudaHirata M, Hayashi YK, Hamada K, Ogata K, Ohno S. 2010. The 8th and 9th tandem spectrin-like repeats of utrophin cooperatively form a functional unit to interact with polarity-regulating kinase PAR-1b. Biochem Biophys Res Commun 391: 812-817.

Yu W, Datta A, Leroy P, O'Brien LE, Mak GZ, Jou TS, Matlin KS, Mostov KE, Zegers MMP. 2005. $\beta 1$-Integrin orients epithelial polarity via Racl and laminin. Mol Biol Cell 16: $433-445$.
Yu W, Shewan AM, Brakeman P, Eastburn DJ, Datta A, Bryant DM, Fan QW, Weiss WA, Zegers MMP, Mostov KE. 2008. Involvement of RhoA, ROCK I and myosin II in inverted orientation of epithelial polarity. EMBO Rep 9: 923-929.

Yurchenco PD. 2011. Basement membranes: Cell scaffoldings and signaling platforms. Cold Spring Harb Perspect Biol 3: a004911.

Yurchenco PD. 2015. Integrating activities of laminins that drive basement membrane assembly and function. Curr Topic Membr 76: 1-30.

Yurchenco P, Cheng Y. 1994. Laminin self-assembly: A threearm interaction hypothesis for the formation of a network in basement membranes. Contrib Nephrol 107: $47-$ 56.

Zuk A, Matlin KS. 2002. Induction of a laminin isoform and $\alpha 3 \beta 1$-integrin in renal ischemic injury and repair in vivo. Am J Physiol Renal Physiol 283: F971-F984. 


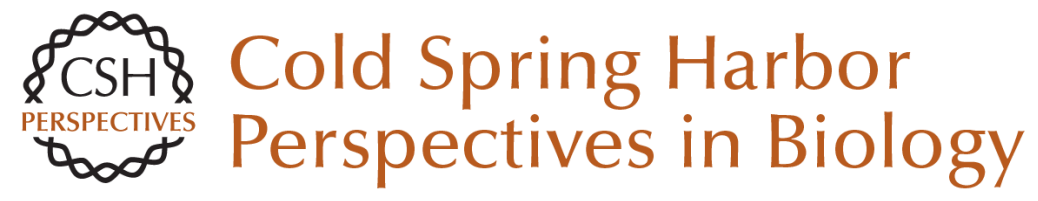

\section{Laminins in Epithelial Cell Polarization: Old Questions in Search of New Answers}

Karl S. Matlin, Satu-Marja Myllymäki and Aki Manninen

Cold Spring Harb Perspect Biol 2017; doi: 10.1101/cshperspect.a027920 originally published online February 3, 2017

\section{Subject Collection Cell Polarity}

Regulation of Cell Polarity by Exocyst-Mediated

Trafficking Noemi Polgar and Ben Fogelgren

Phosphoinositides and Membrane Targeting in Cell Polarity

Gerald R. Hammond and Yang Hong

Trafficking lon Transporters to the Apical

Membrane of Polarized Intestinal Enterocytes Amy Christine Engevik and James R. Goldenring

Signaling Networks in Epithelial Tube Formation Ilenia Bernascone, Mariam Hachimi and Fernando Martin-Belmonte

Making Heads or Tails of It: Cell-Cell Adhesion in Cellular and Supracellular Polarity in Collective Migration Jan-Hendrik Venhuizen and Mirjam M. Zegers

Laminins in Epithelial Cell Polarization: Old

Questions in Search of New Answers

Karl S. Matlin, Satu-Marja Myllymäki and Aki Manninen

Epithelial Morphogenesis during Liver Development

Naoki Tanimizu and Toshihiro Mitaka
The Crumbs3 Polarity Protein

Ben Margolis

Microtubule Motors in Establishment of Epithelial Cell Polarity Geri Kreitzer and Monn Monn Myat

Role of Polarity Proteins in the Generation and

Organization of Apical Surface Protrusions Gerard Apodaca

Polarized Exocytosis Jingwen Zeng, Shanshan Feng, Bin Wu, et al.

Regulation of Transporters and Channels by Membrane-Trafficking Complexes in Epithelial Cells

Curtis T. Okamoto

Membrane Transport across Polarized Epithelia Maria Daniela Garcia-Castillo, Daniel J.-F. Chinnapen and Wayne I. Lencer

Mechanisms of Cell Polarity-Controlled Epithelial Homeostasis and Immunity in the Intestine Leon J. Klunder, Klaas Nico Faber, Gerard Dijkstra, et al.

For additional articles in this collection, see http://cshperspectives.cshlp.org/cgi/collection/

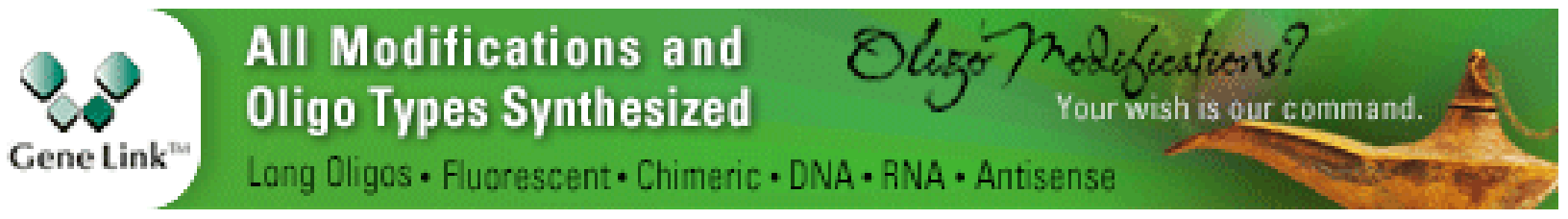


Targeting the Mucosal Barrier: How Pathogens Modulate the Cellular Polarity Network Travis R. Ruch and Joanne N. Engel
The Biology of Ciliary Dynamics

Kuo-Shun Hsu, Jen-Zen Chuang and Ching-Hwa Sung

For additional articles in this collection, see http://cshperspectives.cshlp.org/cgi/collection/

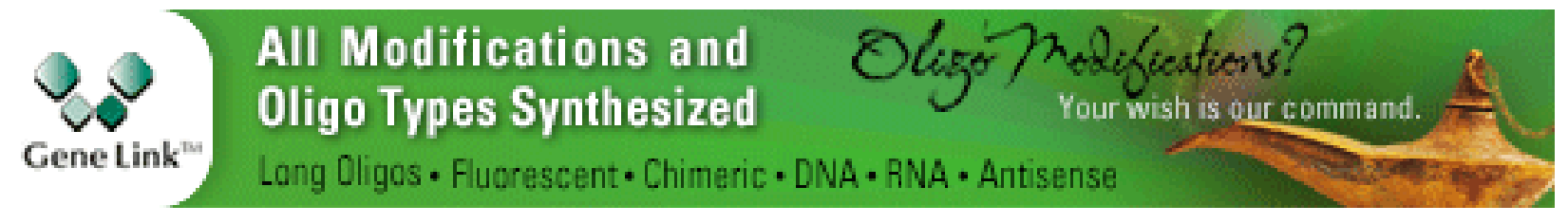

Copyright @ 2017 Cold Spring Harbor Laboratory Press; all rights reserved 